Eur. J. Clin. Chem. Clin. Biochem.

Vol. 29, 1991, pp. 105-110

(C) 1991 Walter de Gruyter \& Co.

Berlin - New York

\title{
A Short Evaluation of a New Haematological Cell Counter - The Cell-Dyn 3000 - Following a Modified Tentative NCCLS-Procedure
}

\author{
By L. van Leeuwen, P. H. Eggels and J. A. Bullen \\ Medische Laboratoria, Sint Jansgasthuis, Weert, The Netherlands
}

(Received May 8/November 9, 1990)

Summary: The proposed procedure offers a method of evaluation that is feasible, but still a very precise method, for the evaluation of electronic differential leukocyte counters. Following this abbreviated method, the classification of the leukocytes, from $5 \times 20$ unselected blood specimens, with a flow-cytometric analysis by the Sequoia Turner Cell-Dyn 3000, was compared with microscopic differential counts as the standard. With proven accuracy and good reproducibility, the Cell-Dyn 3000 is a good sieve for the selection of normal samples. The high sensitivity and fair specificity permits a reduction of the differential workload to about 30 percent of the normal workload.

\section{Introduction}

The U.S. National Committee for Clinical Laboratory Standards (NCCLS) has developed a comprehensive scheme, H20-T (1) for assessing electronic differential leukocyte counters using manual differentiation as the reference. This scheme includes 150 normal and 150 abnormal specimens (differentiation of 800 cells in each) to reduce the subjective and qualitative aspects of the classical single-slide, 100cell differential count (manual 100).

Several evaluations of the safety and efficacy of electronic differential leukocyte counting instrumentation have been used to compare instrument counts with those obtained from 800 cell, multislide and light microscopic differential counts $(2,3)$. The method of evaluation proposed by the NCCLS is cumbersome, laborious and time consuming. Most of the time, evaluation material in middle and small laboratories is available in sufficient amount, but the manpower is limited. Therefore most authors use their own methods (4-7) for comparison, while others make use of the Technicon $\mathrm{H}-6000$ as a new kind of standard $(8-11)$.
Kohut (12) published an abbreviated method (using 20 samples) for assessing leukocyte counters, because the H20-T was laborious and would have taken several months.

We found this method to be concise, and we have chosen it for a 100 specimen-modification of the NCCLS procedure (400 cell, multislide microscopic differential count) for evaluating a new electronic differential leukocyte counter, the Cell-Dyn 3000 from Sequoia-Turner Corp, Mountain View, Ca, U. S. A.

Our aim was to determine whether the Cell-Dyn 3000 could discriminate between a normal and a pathological blood sample, especially with respect to the leukocyte differentiation.

The evaluation was performed in two different ways: first in terms of a "naked" differentiation thus including only the five leukocyte sub-populations in percentages; secondly in terms of a "total" differentiation, regarding percentages and absolute numbers and including all flagging. 


\section{Materials and Methods}

\section{System description}

The CELL-DYN 3000 is a particle counter, sizer and white blood cell classifier, dedicated to the electronic and optical detection and measurement of blood cells contained in a sample of whole blood. EDTA-anticoagulated whole blood is aspirated via either the open or closed sampler.

The electronic resistance. with volumetric metering, is employed to count and size red blood cells and platelets [Coulter 1956]. Haemoglobin is measured by a modified haemiglobin cyanide absorbance method with auto blank.

A laser-based flow cytometer is employed to count, size and classify leukocytes. Simultaneously, during the measurement cycle, each white cell is counted and individually characterized by four specific angles of light scatter for the differential classification of the lymphocytes, basophils, monocytes, neutrophils and eosinophils. This is referred to as Multi-Angle Polarized Scatter Separation $(13-15)$.

The erythrocyte and platelet pulses are collected and presented in numbers per litre whole blood and in frequency distribution curves (histograms). Simultaneously, data are derived for the Red Cell Indices, Red Cell Distribution Width, Mean Platelet Volume and Platelet Distribution Width.

Total leukocyte counts are collected and presented in numbers per litre whole blood.

As stated above, each leukocyte is individually characterized by four scatter pulses. Once the signals of each cell have been placed in their corresponding computer channels, the computer software then multi-dimensionally classifies the signals into the 5-part differential.

The leukocyte differential data can be routinely viewed/displayed in the form of two 2-dimensional scattergrams.

\section{Specimen collection}

Blood samples were taken by venapuncture, using Venoject tubes with potassium $\left(\mathrm{K}_{2}\right)$-EDTA as an anti-coagulant.

Over a period of one week, 20 patient samples were collected each day, thereby providing 100 patient samples. The distribution of our patient population, is normally one third clinical patients, one third polyclinical and one third general practitioner patients.

Every fifth sample was taken into the test procedure until the desired number had been tested, i. e. 6 clinical patients, 6 polyclinical and 6 general practice patients. In an effort to include a wide range of abnormal values, the clinical patients were mostly selected from the internal and paediatric (nucleated erythrocytes) clinic. Two other samples were added with known pathology.

The subjects were predominantly white Caucasian and included 42 males and 58 females; ages ranged from 6 months to 89 years.

\section{Reference method}

Four smears were made from each of these 20 samples, labeled with the patient identification and the characters A, B, C or D. These smears were stained within one hour, using a Wescor Aerospray Slide Stainer. The slides were then randomly distributed to four highly experienced technologists. Each technologist reported a 100 -cell differentiation, thereby producing a 400 -cell count on each specimen. All leukocyte populations were reported in relative percentages, as is usual in a 100-cell differentiation. Flags (both for erythrocytes and leukocytes as for platelets) also were mentioned on the report form.

The slides were stored separately in case a closer review became necessary.

\section{Cell-Dyn 3000 method}

Between half an hour and an hour after sampling, the samples were analysed three times in a different sequence on the CellDyn 3000. Results and histograms were stored with identification, sample status (abnormal or normal), age and sex. The three results from the Cell-Dyn 3000 were then collected on one report form per patient, together, with the dispersional and morphological flags.

\section{Results}

Results for haemoglobin, erythrocytes, leukocytes and platelets were compared with those from the Coulter Counter S5 and the Medonic CA 480. These instruments were adjusted on a regional (15 laboratories) monthly quality control programme with fresh blood samples. The results in the Ortho QC-programme lay within 2 s.d.

For all parameters mentioned above, the correlation coefficient was $>0.98$.

\section{Linearity}

The linearity of the apparatus was evaluated by eight successive percentile dilutions $(100,80,60,40,20,15$, 10 and $5 \%$ ). To cover the whole dynamic range of haematology parameters, the analyses were performed on a normal patient sample, on a buffy coat, on packed cells and on platelet rich plasma. All the components were obtained from one single donor. The average of two duplicate specimens was plotted against the expected value for each dilution percentile.

The values lay well within the manufacturer's specifications.

\section{Carry-over}

The procedure was examined for carry-over with two samples, one with high and one with low haematology parameters. For this purpose, two blood samples were taken from a donor, the first being concentrated, the other diluted to provide respectively high and low haematological parameters.

Independent triplicate cycling was performed on the concentrated sample, followed by a triplicate analysis of the diluted sample. The average sets of the three values were used to calculate the carry-over for haemoglobin, leukocytes; erythrocytes and platelets according to the following formula.

$$
\frac{\mathrm{L} 1-\mathrm{L} 3}{\mathrm{H} 3-\mathrm{L} 3}
$$

Carry-over was not found, except in the case of leukocytes, the deviation being of little or no clinical significance.

Eur. J. Clin. Chem. Clin. Biochem. / Vol. 29, 1991 / No. 2 


\section{Within-run precision}

Within-run precision was evaluated for the directly measured parameters, i. e. leukocytes, leukocyte subpopulations, erythrocytes, haemoglobin, mean corpuscular volume, platelets and the derived parameter haematocrit (tab. 1).

One vial of Ortho Normal-15 Control Blood was aspirated twelve times, and the results were stored in an empty control file. The two first aspirations were deleted as primes and the coefficients of variation (CV) of the ten remaining samples were calculated by the apparatus.

The same performance was completed for a patient sample. The results of these ten samples are presented between brackets.

The precision of the instrument showed a coefficient of variation of less then $1.5 \%$, except for the leukocyte subpopulations.

With fresh human blood, the coefficient of variation was far better (see discussion).

\section{Run-to-run precision}

For five consecutive days a freshly opened vial of Ortho Normal-15 Control Blood was analysed in two replicate analyses. The results were stored in an empty control file and the CV's were calculated (tab. 2).
Influence of the preservation time on the count

Several patient samples were analysed ten times at hourly intervals. Samples were kept at room temperature. All haematology parameters, included the leukocyte differentiation, were examined.

The quality of the instrumental differentiation was not influenced for a normal working day (8 hours).

Evaluation criteria for the leukocyte differential count

We examined the correlation between the differential cell-populations produced by the Cell-Dyn 3000 and the reference method, i.e. lymphocytes vs lymphocytes, neutrophils vs neutrophils, monocytes vs monocytes, eosinophils vs eosinophils and basophils vs basophils.

Accuracy may be defined as the degree of conformity to a true value. When comparing a new method with a reference method, which in this case is regarded as a true value, the intercept, slope and correlation coefficient indicate the accuracy of that new method (fig. $1 \mathrm{a}-\mathrm{d})$.

For a full scale of values from low to high, the CellDyn 3000 scores very well for neutrophils, lymphocytes, eosinophils and less for monocytes. This

Tab. 1. Within-run precision of the Sequoia Turner Cell-Dyn 3000 for Ortho Normal-15 Contol Blood. Between brackets the results for a fresh normal patient sample.

\begin{tabular}{lclll}
\hline Parameter & $\overline{\mathrm{x}}$ & SD & CV \% & Units \\
\hline Erythrocytes & 4.45 & 0.03 & 0.8 & $10^{12} / 1$ \\
Haemoglobin & 8.7 & 0.0 & 0.4 & $\mathrm{mmol} / 1$ \\
Haematocrit & 0.422 & 0.004 & 0.8 & - \\
Mean corpuscular volume & 94.7 & 0.2 & 0.2 & $\mathrm{fl}$ \\
Leukocytes & 4.9 & 0.1 & 2.9 & $10^{9} / 1$ \\
Neutrophils & 56.5 & 1.5 & $2.7(1.8)$ & $\%$ \\
Lymphocytes & 21.7 & 1.3 & $6.0(1.1)$ & $\%$ \\
Monocytes & 0.3 & 0.2 & $(4.4)$ & $\%$ \\
Eosinophils & 21.3 & 0.8 & $3.7(9.4)$ & $\%$ \\
Basophils & 0.2 & 0.2 & $-(6.6)$ & $\%$ \\
\hline
\end{tabular}

Tab. 2. Run-to-run precision of the Sequoia Turner Cell-Dyn 3000 for Ortho Normal-15 Contol Blood.

\begin{tabular}{|c|c|c|c|c|}
\hline Parameter & $\overline{\mathbf{x}}$ & SD & CV $(\%)$ & Units \\
\hline Erythrocytes & 4.48 & 0.08 & 1.7 & $10^{12} / 1$ \\
\hline Haemoglobin & 8.65 & 0.11 & 1.25 & $\mathrm{mmol} / \mathrm{l}$ \\
\hline Haematocrit & 0.42 & 0.007 & 1.7 & - \\
\hline Mean corpuscular volume & 94.5 & 0.56 & 0.60 & n \\
\hline Leukocytes & 4.72 & 9.2 & 1.9 & $10^{9} / 1$ \\
\hline Neutrophils & 57.2 & 1.2 & 6.1 & $\%$ \\
\hline Lymphocytes & 21.7 & 1.4 & 6.5 & $\%$ \\
\hline Monocytes & 0.3 & 0.2 & - & $\%$ \\
\hline Eosinophils & 20.7 & 1.2 & 6.0 & $\%$ \\
\hline Basophils & 0.2 & 0.1 & - & $\%$ \\
\hline
\end{tabular}



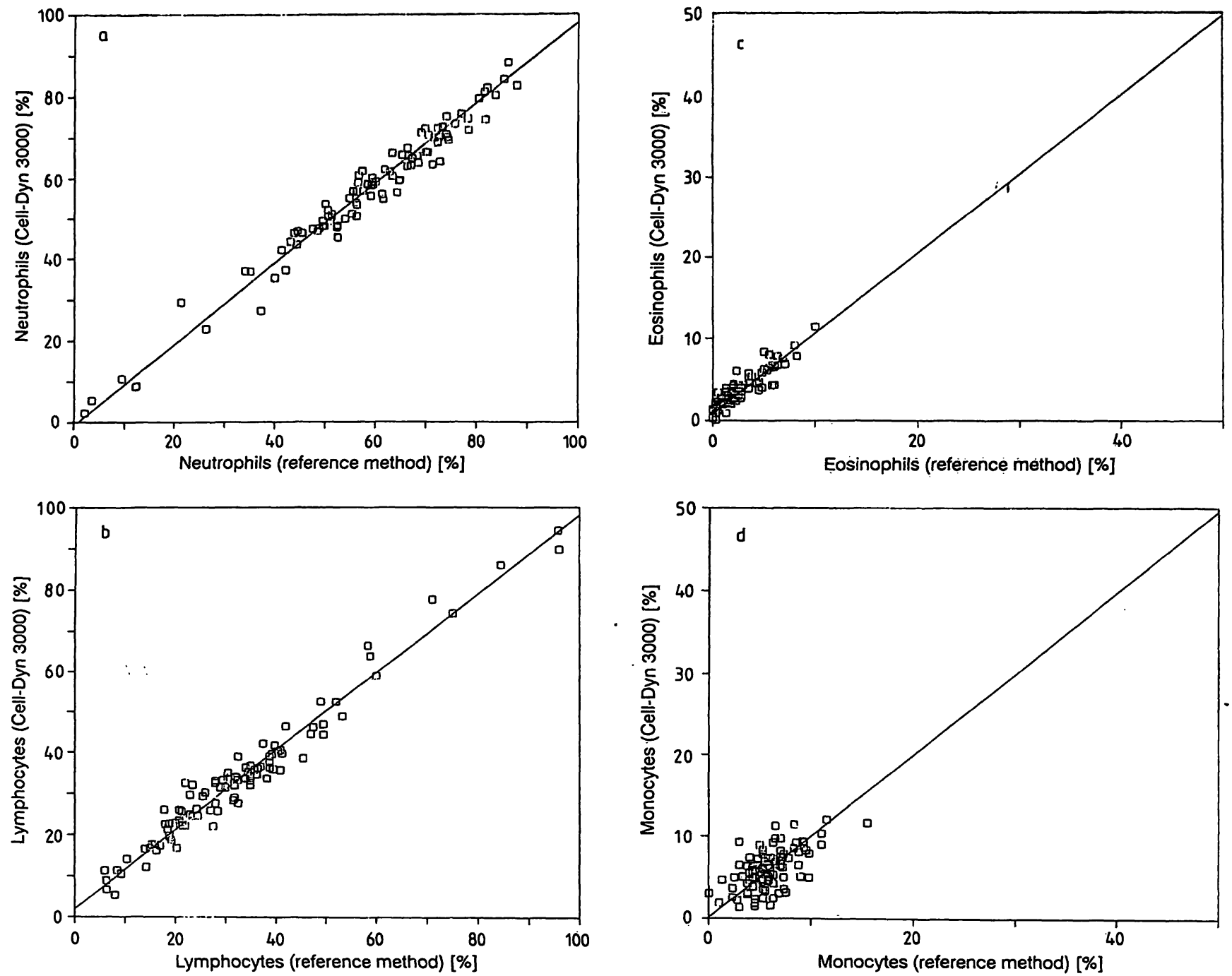

Fig. 1. Orthogonal regression of the Cell-Dyn 3000 versus the 400 -cell manual differentiation

$$
\begin{aligned}
& \text { a. \% Neutrophils } \\
& \text { Intercept }=-0.602594, \text { slope }=0.98482 \\
& r=0.96538 \\
& \mathrm{Sb}=0.026899, \mathrm{Sa}=1.61512, \mathrm{Sy} 0=1.59934 \\
& \mathrm{~b} . \% \text { Lymphocytes } \\
& \text { Intercept }=2.084834, \text { slope }=0.96066 \\
& \mathrm{r}=0.98101 \\
& \mathrm{Sb}=0.01919, \mathrm{Sa}=0.70819, \mathrm{Sy} 0=0.74555
\end{aligned}
$$

c. \% Eosinophils

Intercept $=1.02075$, slope $=0.97890$

$r=0.90981$

$\mathrm{Sb}=0.04513, \mathrm{Sa} \equiv 0.14266, \mathrm{Sy} 0=0.18162$

d. \% Monocytes

Intercept $=0.160106$, slope $=0.99428$

$r=0.60863$

$\mathrm{Sb}=0.134059, \mathrm{Sa}=0.79252, \mathrm{Sy} 0=0.81277$ method, however, is not very useful for the low values of the basophil count. Therefore, we plotted the difference per differentiation against the mean difference, then measured the intercept.

As a control, we also used this techniques on the monocyte and eosinophil population. Values correlated well with the intercept values (tab.3).

Assignment of the classification "normal" or "pathological" was made on the basis of the reference values in use in our laboratory which compare well with the reference ranges mentioned in the literature (16) (tab. 4).
The lower limit for the eosinophils, however, was decreased to " 0 " since low values are of no clinical importance and therefore were catalogized as normal.

\section{Specificity and sensitivity}

Each sample analysed was placed in one of four groups "true normal (TN)", "true pathological (TP)", "false normal (FN)" and "false pathological (FP)". The reference method (manual differentiation) is used as the standard to determine. whether a sample is normal or pathological. 
Tab. 3. Difference per differentiation against the mean difference.

A. Monocytes: Mean difference $=+0.16 \%$

B. Eosinophils: Mean difference $=+1.055 \%$

C. Basophils: Mean difference $=+0.586 \%$.

Tab. 4. Reference interval (normal range) for microscopic blood cell differentiation and for the Sequoia Turner Cell-Dyn 3000. Based on these ranges, the results are classified as "normal" or "pathological".

\begin{tabular}{lll}
\hline & $\begin{array}{l}\text { SI-units } \\
\left(10^{9} / 1\right)\end{array}$ & $\begin{array}{l}\text { Percentages } \\
(\%)\end{array}$ \\
\hline Lymphocytes & $0.8-4.8$ & $20-40$ \\
Neutrophils & $2.0-8.2$ & $50-68$ \\
Monocytes & $0.08-1.2$ & $2-10$ \\
Eosinophils & $0.08-0.6$ & $2-5$ \\
Basophils & $0.0-0.2$ & $0-2$ \\
\hline
\end{tabular}

The following indices were calculated for the CellDyn 3000:

Sensitivity $=\mathrm{TP} /(\mathrm{TP}+\mathrm{FN})$;

Specificity $=\mathrm{TN} /(\mathrm{TN}+\mathrm{FP})$;

Predictive value of true-positive test results among all positive test results $=\mathrm{TP} /(\mathrm{TP}+\mathrm{FP})$;

Predictive value of true-negative test results among all negative test results $=\mathrm{TN} /(\mathrm{TN}+\mathrm{FN})$;

Efficiency $=\mathrm{TP}+\mathrm{TN} /(\mathrm{TP}+\mathrm{FN}+\mathrm{FP}+\mathrm{TN})$.

In both comparisons, microscopic differentiation of the 100 smears revealed $36 \%$ as "normal" and $64 \%$ as "pathological" (tab. 5).

Tab. 5. Evaluation of the analysis of 100 blood samples. Microscopic differentiation is the reference.

1. Differentiation in percentages.

\begin{tabular}{llll}
\hline & & \multicolumn{2}{c}{ Cell-Dyn 3000 } \\
\cline { 3 - 4 } & & Pathological & Normal \\
\hline \multirow{2}{*}{ Reference } & Pathological & 59 & 5 \\
& Normal & 8 & 28 \\
\hline
\end{tabular}

2. "Total" Differentiation.

\begin{tabular}{llcc}
\hline & & \multicolumn{3}{c}{ Cell-Dỵ 3000 } & \\
\cline { 3 - 4 } & & Pathological & Normal \\
\hline Reference & Pathological & 63 & 1 \\
& Normal & 8 & 28 \\
\hline
\end{tabular}

\section{Discussion}

Because of the good linearity (leukocytes up to 99.9 $\times 10^{9} / 1$, erythrocytes up to $\left.7.00 \times 10^{12} / 1\right)$, accuracy and reproducibility, together with the good sensitivity and the fair specificity, the Cell-Dyn 3000 fulfils its purpose as a sieve function. Although the specificity is $77.9 \%$, this has only minor consequences for the total workload. In fact, put to the test, our daily workload in manual differentiations has fallen to $30 \%$ of the total.

In our opinion, the evaluation method has proved to be suitable, providing within one week an evaluation of the possibilities and impossibilities of an instrument.

The results of our study show that the rate of "false normal" results represents only $1 \%$ of the total. The impression exists that an enlargement of the test sample size will give an even better result, because the deviant sample showed only minor differences.

Of 2 microscropically abnormal specimens, which had no flags in the Cell-Dyn, one could be eliminated because of a double population in the erythrocyte graph. The other one showed only negligible differences between the manual and Cell-Dyn values.

Of the eight samples which were abnormal in the CellDyn, seven showed relatively small differences and would normally have been accepted as normal for clinical purposes. Only one was a case of mild monocytosis $(11.5 \%$ against $6.5 \%)$.

This means that the results for diagnostic sensitivity, specificity, efficiency and predictive value are in practice better than the ones showed in table 6 .

Tab. 6. Diagnostic values of the Sequoia Turner Cell-Dyn 3000 results, expressed as the \% probability with the results of the microscopic differentiation.

\begin{tabular}{lll}
\hline & Percentages & Total \\
\hline Diagnostic sensitivity & 92.2 & 98.4 \\
Diagnostic specificity & 77.8 & 77.9 \\
$\begin{array}{l}\text { Predictive value of the } \\
\quad \text { abnormal }\end{array}$ & 88.1 & \\
$\quad$ normal & 84.8 & 88.7 \\
Efriciency & 87.0 & 91.0 \\
\hline
\end{tabular}

A left shift without increased leukocyte count is not always alerted, for example in the case of an extramedullar haemopoesis. 
Although showing an absolute mean difference of $1.055 \%$ in relation to the manual differentiation, the apparatus gives a fair representation of the decrease or increase in eosinophils.

It is also notable that the Cell-Dyn 3000 normally does not count the nucleated red blood cells as leukocytes. In the case of numerous erythroblasts or basophilic normoblasts, however, the Cell-Dyn 3000 fails, because it cannot distinguish these greater cells from lymphocytes. In the case of a massive normoblastosis (ratio: 185 normoblasts to 100 leukocytes) there is an overflow into the lymphocyte count. A massive normoblastosis, however, is for the attentive operator visible as a 'cluster' beneath the lymphocyte population in the scattergram.

In one patient with an infection of Plasmodium falciparum (with ring forms and gametocytes) the apparatus did not recognize the intracellular inclusions. Yet it gave an alert, because of the monocytosis $(25 \%)$.

\section{References}

1. National Committee For Clinical Laboratory Standards: NCCLS Tentative Standard, H20-T: 1984, 4.

2. Breakell, E. S., Marchand, A., Marcus, R. \& Simson E. (1985) Comparison of Performance for Leukocyte Differential Counting of the Technicon H6000 System with a Manual Reference Method Using the NCCLS Standard. Blood Cells 11, 257-279.

3. Sallitt, R. L., Ho, T. T. \& Rodriguez, R. R. (1985) Evaluation of Leukocyte Differential Counts on the QBC Centrifugal Hematology Analyzer According to NCCLS Standard H20-T. Blood Cells 11, 281-294.

4. Buergi, W. \& Marti, H. R. (1989) Automated Blood Count Analysis by Trimodal Size Distribution of Leukocytes with the Sysmex E-5000. J. Clin. Chem. Clin. Biochem. 27, 365368.

5. Ennen, J., Bauerle, R. \& Schriewer, H. (1990) Vergleich der Bestimmung von Leukozytenpopulationen am Coulter VCS mit der Mikroskopischen Differenzierung. Lab. Med. 14, $102-110$.

6. Verbruggen, H. W., Wessels, J. M. C., Boezeman, J. B. M. \& Haanen, C. (1989) Vergelijking van de microscopische leukocytendifferentiatie met de geautomatiseerde leukocytendifferentiatie op de HI. Tijdschr. NVKC 14, 109-115.

7. Thomas, L., Mueller, Th. \& Rodrian, J. (1989) Vergleich der Bestimmung von Leukozytenpopulationen am Technicon $\mathrm{Hl}$ mit der mikroskopischen Differenzierung. Lab. Med. 13, 213-219.

8. Smeets, E. H. J. \& van Wersch, J. W. J. (1988) Performance of a Three-part Dif Impedance Cytometer (Sysmex E-4000) in Comparison with a Cytochemical Cytometer (Technicon H-6000). J. Clin. Chem. Clin. Biochem. 26, 531-540.
We have also noticed that the control blood in use was not suitable for instrumental differentiation with the Sequoia Cell-Dyn 3000. This was the case with all Impedance Control Blood. With fresh human blood, the coefficient of variation for the leukocytes and leukocyte subpopulations was far better (tab. 1). This is probably due to the fact that living leukocytes do not shrink while being counted in the Cell-Dyn 3000 .

To summarize, we have found that the Sequoia Turner Cell-Dyn 3000 is a reliable and accurate differential cell counter. The fact that the Cell-Dyn 3000 uses the leukocytes without stripping, lysing, colouring, etc., gives us much more confidence in the results.

Moreover, the use of physical measuring principles for all the determinations, means that the instrument is mechanically robust with an easy-to-perform maintenance.

9. Wielders, J. P. M., Beunis, M. H. \& van Wersch, J. W. J. (1986) A Comparison of the Screening Ability of Two Automated Leukocyte Differential Counters. J. Clin. Chem. Clin. Biochem. 24, 472-480.

10. Katz, N. \& Lenz, T. (1988) Vergleich der Volumen-Verteilungs-Analyse von Leukozyten am Coulter Stacker mit der cytochemisch-cytometrischen Klassifizierung am Technicon H 6000 und der mikroskopischen Differenzierung. Lab. Med. 10, 354-362.

11. van Wersch, J. W. J. \& Bank, C. (1990) A New Development in Haematological Cell Counting: The Sysmex NE-8000, Automaton for Cell Count and Physical Five-Part Leukocyte Differentiation. J. Clin. Chem. Clin. Biochem. 28, 233-240.

12. Kohut, M. L. (1989) An Abbreviated Method for Assessment of Electronic Differential Leukocyte Counters. Labmedica, Vol VI, 4, 27-34.

13. Salzmann, G. C., Growel, J. M. \& Martin, J. C. (1975) Cell Classification by Laser Light Scattering. Identification and Separation of Unstained Leukocytes. Acta Cytol. 19, 374377.

14. Terstappen, L. W. M. M., de Grooth, B. G., Visscher, K., van Kouterik, F. A. \& Greve, J. (1987) Four-Parameter White Blood Cell Differential Counting Based on Light Scattering Measurements. Cytometry 9, 39-43.

15. de Grooth, B. G., Terstappen, L. W. M. M., Puppels, G. J. \& Greve, J. (1987) Light-Scattering Polarization Measurements as a New parameter in Flow Cytometry. Cytometry 8, 539-544.

16. Wintrobe, M. M. (1981) Clinical Hematology. 8th edn., p. 1888 , Lea \& Febiger, Philadelphia.

Drs. L. van Leeuwen

Medische Laboratoria

Sint Jansgasthuis

Vogelsbleek 5

NL-6001 BE Weert 